\title{
Gestión del riesgo y política pública en el cantón de Desamparados, Costa Rica
}

Risk assessment and public policy in the canton of Desamparados, Costa Rica

Gestão do risco e política pública no bairro de Desamparados, Costa Rica

Adolfo Quesada-Román

adolfo.quesada@gmail.com

Escuela de Ciencias Geográficas,

Universidad Nacional.

Heredia, Costa Rica.

Orcid: https://orcid.org/0000-0001-6601-5254

\section{Guillermo Calderón-Ramírez}

gcalderon2809@gmail.com

Escuela de Ciencias Geográficas

Universidad Nacional.

Heredia, Costa Rica.

Orcid: https://orcid.org/0000-0003-0830-3164

Recibido-Received: $\mathbf{5 / d i c / 2 0 1 6} \bullet$ Corregido-Corrected: $\mathbf{2 9 / a b r / 2 0 1 7}$

Aceptado-Accepted: 10/set/2017 • Publicado-Published: 31/jul/2018

\section{Resumen}

Desamparados es uno de los cantones de Costa Rica más afectados por los riesgos naturales, según datos registrados de los últimos 45 años. Sus características físico-geográficas y socioeconómicas, así como el desarrollo urbano sin una planificación territorial efectiva generan mayor presión para contribuir al riesgo de desastre. Esta unidad político-administrativa es un verdadero reto para la gestión del riesgo de desastres y el ordenamiento territorial desde la política pública, ya que mezcla una serie de paisajes rurales y urbanos, además del impacto recurrente de deslizamientos e inundaciones con un marco jurídico entrabado para solventar estas problemáticas socioambientales.

Palabras clave: riesgos naturales; desastres; vulnerabilidad; ordenamiento territorial; Desamparados

\section{Abstract}

Desamparados is one of the counties most affected by natural hazards each year in Costa Rica during the last 45 years. Their physical-geographical and socioeconomic characteristics, urban development without effective land use planning on risk areas generate economic and human losses. This political-administrative unit is a real challenge to manage disaster risk and land use planning from public policy as it mixes a number of rural and urban landscapes, in addition to the recurring impact of landslides and floods with hindered legal framework for address these social and environmental problems.

Keywords: natural hazards; disasters; vulnerability; land use planning; Desamparados 


\section{Resumo}

Desamparados é um dos municípios da Costa Rica mais afetados pelos riscos naturais, segundo dados registrados dos últimos 45 anos. Suas características físico-geográficas e socioeconômicas e também o desenvolvimento urbano sem uma planificação territorial efetiva geram maior pressão no favorecimento de risco de desastre. Esta unidade político-administrativa é um verdadeiro desafio para a gestão do risco de desastres e do ordenamento territorial desde a política pública, já que mescla uma serie de paisagens rurais e urbanas, além do impacto incessante de deslizamentos e inundações com um marco jurídico travado para solucionar estas problemáticas socioambientais.

Palavras-chave: riscos naturais; desastres; vulnerabilidade; ordenamento territorial; Desamparados

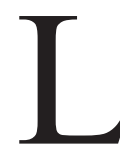

a gestión del riesgo ha evolucionado con el pasar de los años, involucrándose una diversidad de agentes económicos, políticos, naturales y sociales. Un medio para solventar la situación del riesgo a desastre es llevado a cabo con la política pública. El llamado a instituciones e investigadores a discutir sobre el tema del riesgo al desastre ha generado avances significativos sobre las razones que lo generan, ocasionando que el Gobierno adopte medidas para prevenir la complejidad de situaciones que los desastres ocasionan. En la gestión del riesgo hay que considerar distintos escenarios, desde la desarticulación y limitación del funcionamiento de los agentes políticos hasta la insistencia y necesidad de los agentes sociales. Para conocer más a fondo el tema en discusión, es importante resaltar que Costa Rica resulta de una configuración tectónica compleja relacionada con los procesos de subducción entre las placas Cocos y Caribe, la colisión de la Serranía o cordillera volcánica submarina de Cocos al SE del país, la unión triple de las placas Cocos-Caribe-Nazca y un activo sistema de fallas transcurrentes asociado con la placa de Panamá que tiene implicaciones directas e indirectas en la sismicidad regional, y el vulcanismo (DeMets et al., 1990; Bird, 2003; Denyer et al., 2003). A su vez, el país está dividido en dos vertientes: Pacífica y Caribe, lo cual favorece la distinción climática con precipitación constante todo el año (Caribe) y dos picos de lluvias con una marcada época seca en la vertiente pacífica (Taylor y Alfaro, 2005). Esta dinámica endógena y exógena activa mantiene condiciones favorables para el desarrollo tanto de fuertes precipitaciones como de deslizamientos e inundaciones que afectan año tras año a la población.

Dado que el país está expuesto a lluvias extraordinarias durante la época lluviosa, así como afectado indirectamente por el impacto de ciclones tropicales tanto del mar Caribe como del océano Pacífico, estos detonantes pueden generar procesos de ladera e inundaciones en regiones de montaña o planicies (Alfaro y Quesada-Román, 2010). Las inundaciones y deslizamientos son los desastres más comunes en Costa Rica y concentran aproximadamente el $90 \%$ de los reportes de desastres desde 1970, además representan entre el $1 \%$ y el $2 \%$ del producto interno bruto (PIB) del país en pérdidas económicas que el Estado debe invertir en reconstrucción, y genera en promedio 15 muertos por año desde 1970 (Quesada-Román, 2015, LA RED, 2016). 
E1 $60 \%$ de la población de Costa Rica se concentra en el Gran Área Metropolitana (GAM), donde se reúnen las opciones laborales, de estudios, de atención médica y de servicios. Esta región geográfica tuvo un crecimiento desmedido desde la década de 1970, haciendo pasar al país de una marcada ruralidad hacia una clara tendencia urbana en esta desordenada metrópoli que aglutina, además de las cuatro ciudades más grandes del país (San José, Alajuela, Heredia, y Cartago), a prácticamente una veintena de cantones a sus alrededores. Del mismo modo, los registros de desastres se ubican, en mayor medida, dentro de la GAM, donde cabe resaltar que, en 45 años de estudio de desastres en esta unidad político-administrativa, se observó cómo el cantón de Desamparados, entre 1985 y 2013, tiene el registro más alto de eventos (Quesada-Román, 2015).

Desamparados es el cantón número tres de la provincia de San José, se localiza al sur de la Gran Área Metropolitana, en la actualidad es uno de los municipios con mayor cantidad y densidad poblacional del país, a causa de una intensa inmigración urbana y rural en las últimas tres décadas. Su fundación se remonta a 1862, y está conformado por 13 distritos: Desamparados, San Miguel, San Juan de Dios, San Rafael Arriba, San Antonio, Frailes, Patarrá, San Cristóbal, Rosario, Damas, San Rafael Abajo, Gravilias y Los Guido. Esta unidad política-administrativa limita al norte con Alajuelita, San José, Curridabat y La Unión; al oeste con Aserrí; al sur con León Cortés y Dota; al este con Cartago y el Guarco (Mapa 1). El cantón de Desamparados presenta un arreglo tectónico, geológico y geomorfológico complejo, que le imprime características geodinámicas intensas, tanto internas como externas, debido al origen, evolución, morfologías y dinámicas del relieve actual, condiciones que hacen de este territorio un espacio geográfico idóneo para el desarrollo de procesos naturales que podrían ser peligrosos para la población.

Esta unidad territorial podría ser dividida, de acuerdo con sus características fisiográficas en las planicies y rampas, así como en las zonas montañosas. Las primeras se asocian con los afluentes que drenan en la depresión Tectónica Central, la cual limita al norte y este con la cordillera Volcánica Central, una sección al sur con los montes del Aguacate y al sur con la cordillera de Talamanca, la cual tiene una de sus estribaciones en las zonas montañosas que posee Desamparados al sur, compuestas de dioritas, granodioritas, cuarzos, gabros y granitos (Vargas, 2012). La precipitación media anual varía entre los 1500 y $3000 \mathrm{~mm}$ anuales (IMN, 2008). No obstante, debido a su localización frente a una serie de cuencas hidrográficas que drenan hacia el río Virilla al norte y el río Candelaria hacia el sur, en eventos de lluvias extraordinarias dichos sistemas fluviales pueden sobrepasar su capacidad de drenaje y provocar inundaciones en comunidades cercanas a dichos cauces. A estas características debe sumarse la condición de fuerte pendiente en las zonas montañosas, lo que incide en la ocurrencia de deslizamientos prácticamente todos los años.

\section{Metodología}

Se genera una revisión bibliográfica sobre los impactos que ha tenido el cantón de Desamparados mediante la información sistematizada por la base de datos de DesInventar, entre 1970 y 2014; este insumo mantiene un registro de desastres ocurridos en América Latina y otras zonas del mundo, el cual permite consultar a escala de municipio los eventos pasados e incluso 
Mapa 1. Localización de Desamparados en su contexto regional

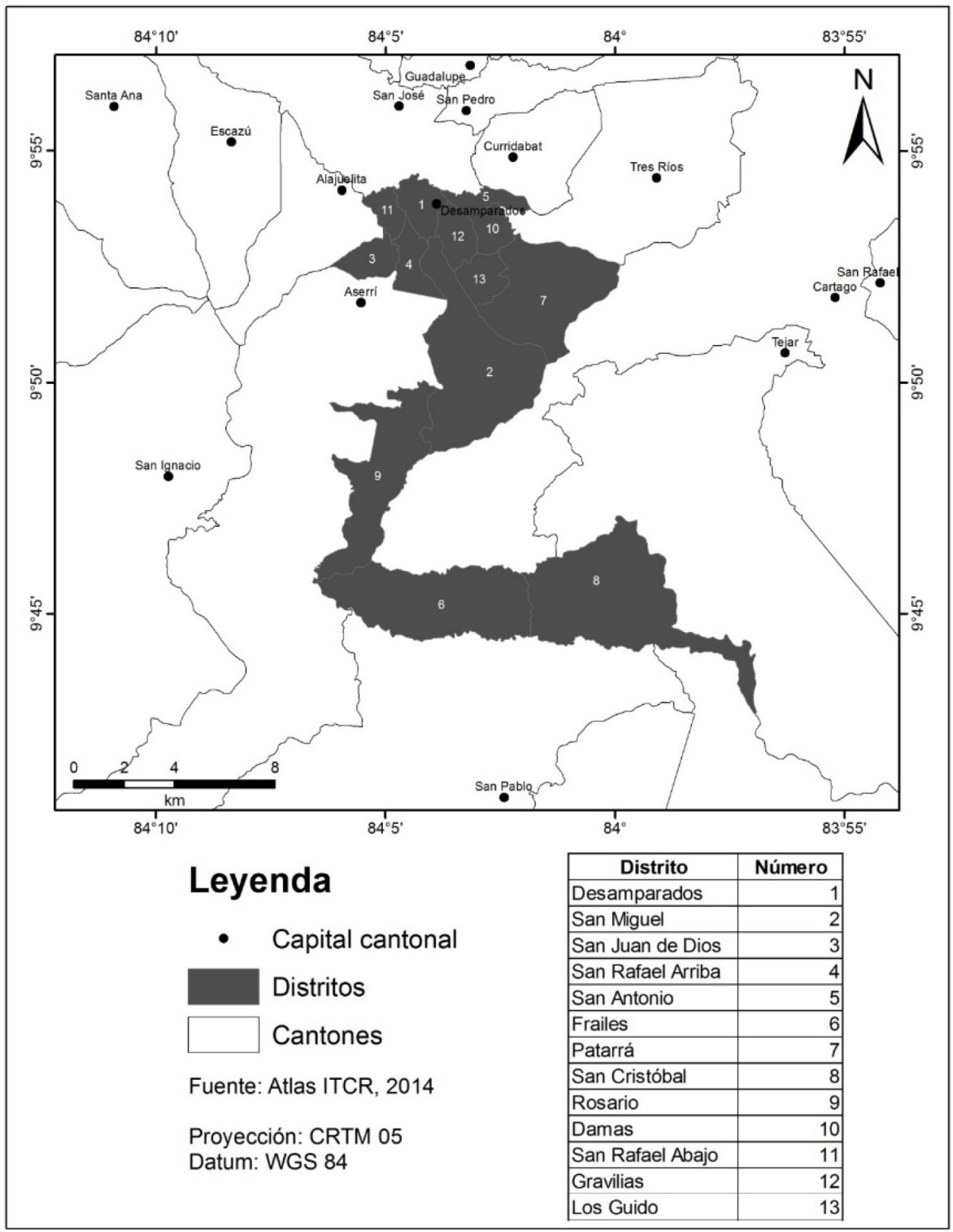

está disponible en el nivel de distrito para Costa Rica (LA RED, 2016). Una vez obtenidos estos datos, son procesados para determinar cuáles fueron las amenazas naturales más recurrentes, los distritos más afectados y los periodos anuales con mayores incidencias de desastres durante 44 años. Para fa- cilitar el análisis de las amenazas naturales, se genera un mapa de regiones morfológicas que explica buena parte de la geodinámica interna y externa del cantón, por medio del cual se logra determinar la incidencia de las amenazas naturales que afectan a la población de esta unidad político-administrativa. 
Por otro lado, se analizan algunas de las diferentes variables socioeconómicas que determinan la vulnerabilidad del cantón de Desamparados, como lo son la pobreza (INEC, 2011), sus índices de desarrollo humano (PNUD, 2007, 2010, 2011) y social (MIDEPLAN, 2013), aumento poblacional (CCP-UCR, 2014), densidad demográfica (CCP-UCR, 2014) y el cambio en el uso de la tierra de espacios rurales hacia zonas urbanas sin un ordenamiento territorial efectivo. El estudio de las amenazas naturales y las vulnerabilidades del cantón podrán dar un panorama más claro de las condiciones de riesgo que predominan en Desamparados y por qué es el cantón más afectado por eventos de desastres en las últimas cinco décadas.

De manera paralela, se hace un análisis de las políticas públicas a escala nacional y municipal, para entender su correlación con la gestión del riesgo de desastres en el municipio de Desamparados a lo largo del periodo en análisis. Para ello fue necesaria una investigación de documentos hemerográficos, leyes decretadas y acciones administrativas tomadas por parte del Gobierno Central de Costa Rica, sus instituciones públicas relacionadas con la temática y la Municipalidad de Desamparados. Por último, se realiza un análisis cruzado de los mecanismos y herramientas políticas más representativos en la gestión del riesgo de desastres desde 1970 en Costa Rica y Desamparados, que pudieran tener alguna injerencia en la mejora de los escenarios de riesgo actuales; para ello, se requirió de una búsqueda bibliográfica e integración de toda la legislación existente.

\section{Resultados}

\section{Marco físico-geográfico}

Para entender la geodinámica interna y externa de Desamparados se hace necesaria una interpretación geomorfológica a grandes rasgos de las regiones morfológicas que componen este territorio. Se realizó un análisis morfométrico y morfológico previo para determinar que este municipio cuenta con tres grandes zonas: planicies y rampas, premontañas y montañas (Mapa 2). Las planicies y rampas se localizan en el cambio de pendiente que se dibuja entre la transición de las montañas y premontañas hacia las partes bajas, asociadas con la depresión Tectónica Central, sector que es drenado por ríos como el Damas, Cucubres y Jorco. Es importante recalcar que esta zona es donde se concentra mayor población que podría verse afectada por procesos de inundación, dadas las bajas pendientes y la alta vulerabilidad tanto física como socioeconómica de estos distritos.

En la vertiente sur que definen las estribaciones montañosas de la Cordillera de Talamanca, de edad miocénica, se observa un denso sistema fluvial caracterizado en el segmento sureste por los ríos San Cristóbal Norte y San Cristóbal Sur, que forman el río Santa Elena y a su vez se unen con los ríos Chirogres, Conejo y Alumbre para formar un segmento del río Grande de Candelaria. En estas zonas dominan las montañas y premontañas donde los procesos de ladera e inundaciones son frecuentes, no obstante, la densidad poblacional es baja. 
Mapa 2. Regiones morfológicas del cantón de Desamparados

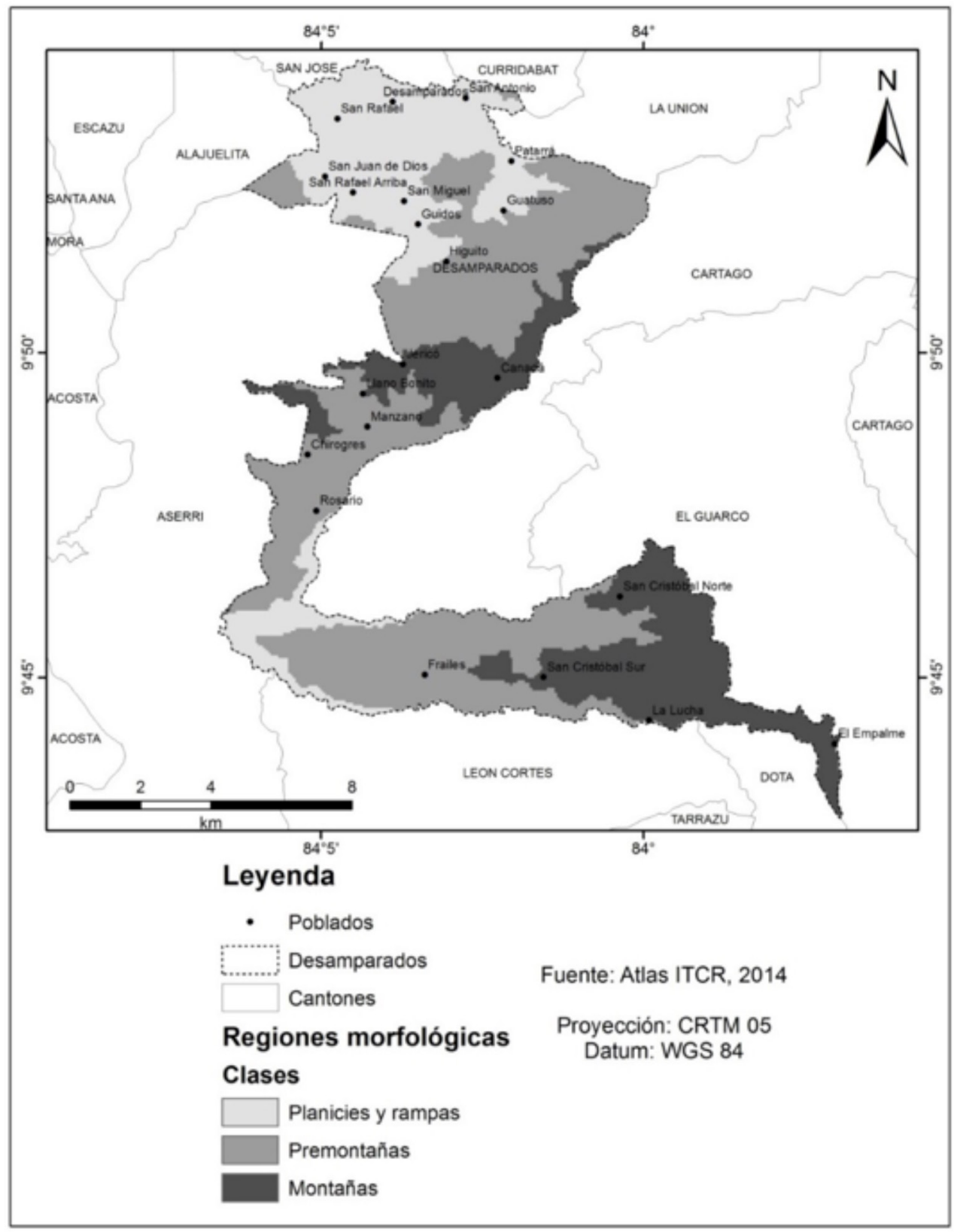

\section{Caracterización socioeconómica}

El cantón de Desamparados está ubicado en la Región Central. Según en el Plan Regional de Desarrollo 2030 desarrollado por el Ministerio de Planificación Nacio- nal y Política Económica (MIDEPLAN), se contempla que el $66 \%$ de la población nacional se va a concentrar en un territorio que comprende el $16 \%$ del país. El MIDEPLAN señala que en el cantón de Desamparados su población supera los 200000 habitantes 
en la actualidad. Los datos que se reflejan en el Cuadro 1 proporcionados por el Atlas del Desarrollo Humano Cantonal, elaborado por el Programa de las Naciones Unidas para el Desarrollo (PNUD) y la Universidad de Costa Rica (UCR), indican que el cantón en estudio, en relación con el Índice de Desarrollo Humano (IDH), el cual analiza tres variables descritas (salud, educación y renta), presentaba un índice de 0,689 en 1992. Posteriormente, para el 2000, ocupó el lugar 29 de los 81 cantones, desplazándose en el quinquenio siguiente a la posición 37; aunque su índice aumentó, la comparación con los demás cantones lo hicieron bajar de posición con respecto a la totalidad del país. Para el 2009, decayó de manera drástica a la posición 70, logrando una recuperación significativa en el 2014 que lo hizo ocupar la casilla 49 en el nivel nacional.

El IDH tal y como menciona Calderón-Ramírez, 2015 para el caso costarricense se mide a través de la salud y es caracterizado por las personas que viven una vida larga y saludable; la educación se basa en la tasa de alfabetización de personas adultas, además de la tasa de matrícula en primaria y secundaria, y la tercera variable analiza la renta, medida por medio del consumo eléctrico residencial per cápita. Aunque la medición de dicho índice podría ser cuestionada, no es el punto por discutir en el presente documento y sí la relación de este con la gestión del riesgo de desastres. Como muestra el Cuadro 1, el IDH establece que cercano a uno es alto, por ende, el cantón de Desamparados se posiciona en números preocupantes, si se compara con el IDH en el nivel de Costa Rica, siendo más vulnerable su población en caso de algún evento de riesgo de desastre. Lo anterior se reitera con la información del MIDEPLAN (2012), señalando que el cantón de Desamparados, en el 2011, presentaba un 17,3\% de hogares en condición de pobreza, así que se revela en un $4,0 \%$ la extrema pobreza. En términos de necesidades básicas insatisfechas (NBI), las cuales miden las condiciones de acceso a vivienda, salud, educación y renta, el Instituto Nacional de Estadística y Censos apunta a que la cantidad de hogares que no presentan acceso a esta condición en el 2011 era de $19,4 \%$, traducida a 11393.00 hogares y a una población de 45252.00 personas.

Por otro lado, el Índice de Desarrollo Social (IDS) se conceptualiza en términos de que la población tenga posibilidades de acceder y disfrutar de un conjunto de derechos básicos, que se agrupan en cuatro dimensiones: económica, participación social, salud y educativa; Desamparados se ubica en la posición 24 a escala cantonal y sus distritos se localizan entre el quinto y tercer quintil, lo que se asocia con buen acceso a las distintas

Cuadro 1. Índice de Desarrollo Humano de Desamparados

Índice de Desarrollo Humano

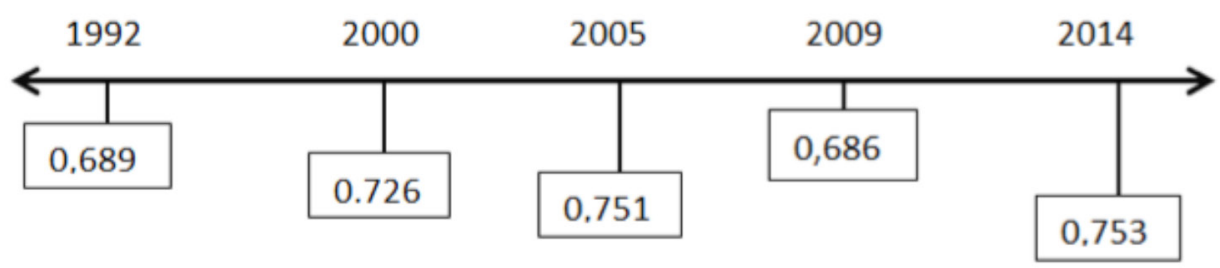

Fuente: PNUD y UCR (2011). 
dimensiones sociales que el índice calcula (MIDEPLAN, 2013). Con lo anterior, hay que colocar atención si se analiza el IDS con los datos demográficos, considerando que, según los censos de población del Instituto Nacional de Estadística y Censo (INEC), se refleja un crecimiento poblacional en los últimos 40 años, prácticamente triplicado, lo que conllevaría tener deficiencias en desarrollo social, si no se contempla ese crecimiento de su población. La mayor importancia de crecimiento poblacional se da entre los años 1984 y 2000, aumentando en 84 654.00 nuevas personas (Cuadro 2).

Como se verá más adelante, los distritos que registran, entre 1970 y 2014, la mayor cantidad de eventos de desastre son estrictamente los que reportan mayor cantidad de población; es decir, los casos de Des- amparados, San Miguel y Los Guido, este último declarado distrito en el 2003, segregado del distrito de Patarrá.

Diagnóstico de la política pública de la gestión del riesgo

En el presente artículo se trabaja con la política pública como aquella opción que tiene la potestad de reducir o prevenir los desastres. Souza (2006), parafraseando a Mead (1995), define política pública “como un campo dentro del estudio de la política que analiza al gobierno a la luz de grandes asuntos políticos" (Souza, 2006, p. 24). Esta misma autora señala que política pública es comprendida como “...el campo del conocimiento que busca, al mismo tiempo colocar al gobierno en acción y/o analizar dicha ac-

Cuadro 2. Población por distrito del cantón de Desamparados

\begin{tabular}{|c|c|c|c|c|}
\hline \begin{tabular}{l}
\multicolumn{2}{c}{ Año } \\
Nombre del dis- \\
trito
\end{tabular} & 1973 & 1984 & 2000 & 2011 \\
\hline Damas & 4376 & 7351 & 12993 & 13175 \\
\hline Desamparados & 30659 & 43352 & 36437 & 33866 \\
\hline Frailes & 2491 & 2622 & 3504 & 3772 \\
\hline Gravilias & - & - & 15846 & 15024 \\
\hline Los Guido & - & - & - & 24102 \\
\hline Patarrá & 1971 & 3390 & 28451 & 11921 \\
\hline Rosario & 1548 & 1606 & 2710 & 3088 \\
\hline San Antonio & 5147 & 7519 & 9775 & 9727 \\
\hline San Cristóbal & 2137 & 2286 & 3360 & 3905 \\
\hline San Juan de Dios & 6465 & 9540 & 16577 & 19481 \\
\hline San Miguel & 8171 & 12053 & 28336 & 31805 \\
\hline San Rafael Abajo & 5757 & 11555 & 22481 & 23283 \\
\hline San Rafael Arriba & 5550 & 7550 & 13008 & 15262 \\
\hline Total & 74272 & 108824 & 193478 & 208411 \\
\hline
\end{tabular}

Fuente: Datos del Instituto Nacional de Estadística y Censos (2016). 
ción (variable independiente) y, cuando fuese necesario, proponer cambios en el rumbo de esas acciones (variable dependiente)" (p.26). El hablar de política pública en un plano general y abstracto debe contener las estructuras de poder y de dominación, así como posteriores conflictos a los que la sociedad se enfrenta y que encuentran en el Estado un lugar para ser administrados (Azevedo, 1997; Calderón, 2015).

El tema de la política es de gran importancia en estudios geográficos, debido a que esta se encuentra presente en todo ámbito. El análisis político debe ir estrechamente ligado a los estudios técnicos, dado que estos son los que lo valoran. Al contar con datos técnicos se logra la valoración de la política, si esta ya se encontrara implementada o, en el caso de no existir, darán criterios para desarrollarla. El enfoque abordado en este documento intenta describir el ordenamiento territorial costarricense y sus deficiencias. Además, se asocia con la gestión del riesgo de desastres en el cantón de Desamparados, debido a las características geomorfológicas y sociales que este presenta.

Los planes reguladores cantonales son caracterizados por tener la autoridad de ejecutar las distintas directrices para el ordenamiento territorial, constituyéndose la principal herramienta con la que los gobiernos locales pueden lograr un territorio equilibrado, y en donde estos pueden ser actualizados cada cinco años. La complejidad en el proceso de trámites, tal y como señalan Ramírez y Villalobos (2014), ha sido causante de atrasos e incluso suspensión en el proceso en el que muchas municipalidades desean contar con un plan regulador. Apegados a lo que indican estos autores, se hace notar que la tramitología institucional-estatal en el desarrollo de los planes reguladores requiere un mayor esfuerzo que va más allá de un análisis técnico ambiental, social o económico para el bienestar de los cantones y su población.

En ordenamiento territorial, Costa Rica mostraba grandes deficiencias, ya que antes del 2012 no contaba con una política de esta índole que fuese integradora, pero es de resaltar que, desde la década de 1950, ya se trataban algunos temas, solo que con una visión meramente urbana, como es el caso de la Ley de Planificación Urbana (4220), creada en 1968. Con el pasar de los años', exactamente en la década de 1990, con la Ley Orgánica del Ambiente se integra lo urbano con lo rural. Para octubre del 2012, se oficializa, por medio del decreto 37623-PLAN-MINAET-MIVAH, la Política Nacional de Ordenamiento Territorial 2012 a 2040; en el Cuadro 3 se sintetiza esta información.

\section{Gestión del riesgo en Costa Rica}

La gestión del riesgo de desastres, según la Estrategia Internacional para la Reducción de Desastres (EIRD) (2016), se define como aquellas decisiones de carácter administrativo, de organización y conocimientos operacionales que desenvuelve la sociedad para definir políticas, con sus estrategias, y fortalecer su capacidad, todo ello para disminuir el impacto de amenazas de carácter natural, desastres ambientales y tecnológicos; importante resaltar que lo mencionado debe incluir medidas para prevenir y mitigar los desastres.

La gestión del riesgo de desastres se maneja en Costa Rica con la Ley Nacional de Emergencias y Prevención del Riesgo (8488) y, junto con ella, el Plan de Gestión del Riesgo constituye el marco estratégico para la aplicación de dicha política. Este plan lo que busca es poner en práctica la política de gestión del riesgo. La política por sí 
Cuadro 3. Instituciones e instrumentos utilizados en ordenamiento territorial

\begin{tabular}{|c|c|}
\hline Instituciones & Instrumentos y atribuciones \\
\hline $\begin{array}{l}\text { Mivah, INVU, Prugam, } \\
\text { municipalidades }\end{array}$ & $\begin{array}{l}\text { Planes reguladores urbanos, Ley de Planificación Urbana } 4240 \\
(1968) \text {, Prugam (2008) / Plan GAM (2013) y lineamientos urbanos. }\end{array}$ \\
\hline $\begin{array}{l}\text { Setena, ICCA, Sinac, } \\
\text { Minae, ICE, ESPH, } \\
\text { Senara }\end{array}$ & $\begin{array}{l}\text { Manejo de cuencas, Grúas II (2004), corredores biológicos, planes } \\
\text { de manejo, Ley Orgánica del Ambiente } 7554 \text { (1995), Ley Forestal } \\
7575 \text { (1996), Ley de Biodiversidad } 7788 \text { (1998), aguas protegidas, } \\
\text { viabilidad ambiental y protección de acuíferos. }\end{array}$ \\
\hline $\begin{array}{l}\text { MAG, INTA, INA, } \\
\text { Senara }\end{array}$ & $\begin{array}{l}\text { Dictamen de fincas, Ley de Uso, manejo y conservación de suelos } \\
7779 \text { (1995), capacidad de uso de la tierra y distribución de tierras. }\end{array}$ \\
\hline MOPT & Planificación y desarrollo de infraestructura estratégica. \\
\hline $\begin{array}{l}\text { CNE, Ministerio de la } \\
\text { Presidencia }\end{array}$ & $\begin{array}{l}\text { Zonas de riesgo, mapas de amenazas naturales y Ley Nacional de } \\
\text { Emergencias y Prevención del Riesgo } 8488 \text { (2005). }\end{array}$ \\
\hline $\begin{array}{l}\text { MIDEPLAN, Cimat, } \\
\text { ICT. }\end{array}$ & $\begin{array}{l}\text { Ley Zona Marítimo Terrestre } 6043 \text { (1977), planes reguladores cos- } \\
\text { teros y Plan Nacional de Turismo Sostenible }(2010-2016)\end{array}$ \\
\hline
\end{tabular}

sola no puede ejecutarse, necesita de medios para lograr desarrollarse y deben ser sometidos a valoración, dado que no siempre cumplen con las expectativas deseadas, ya sea por la formulación de esta o su aplicación metodológica. En el caso de Costa Rica, la política nacional en relación con la gestión del riesgo contempla como área de acción la atención y prevención del riesgo, en la que convergen instituciones como la Comisión Nacional de Emergencias y el Ministerio de la Presidencia, las cuales cumplen dicha función teniendo como instrumentos y atribuciones los mapas de amenazas, la Ley Nacional de Emergencias, el Plan Nacional de Emergencias y las zonas de riesgo.

Para el análisis de la gestión del riesgo de desastres se toma como parámetro el gobierno local del área de estudio. Para este caso, el ente responsable directo debe ser la municipalidad del cantón de Desamparados, la cual no cuenta con una oficina que se encargue de tal gestión. La situación de Desamparados es alarmante, ya que dicho cantón no solo registra la mayor cantidad de amenazas naturales en el país, sino que es uno de los cantones más urbanizados y poblados.

Es prudente valorar la función que deben asumir los gobiernos locales, en primera instancia, para lograr una eficiente gestión del riesgo de desastres. Es preciso considerar que toda política debe ser formulada con base en estudios técnicos, esto da un alto grado de confiabilidad y, consecuentemente, de efectividad a la hora de implementarla. Con todo esto, en temas legales, lo que se acerca más a la realidad sobre el tema es la Ley Nacional de Emergencias y Prevención del Riesgo (8488). En el capítulo II, artículo 5, se habla sobre política de gestión del riesgo, con la cual se explica tanto que dicho tema es de importancia estatal como que debe articular instrumentos, programas y recursos públicos, con el único fin de evitar que ocurran desastres y de atender las emergencias. 
Análisis del riesgo en el cantón de Desamparados

Para desarrollar un análisis del riesgo del cantón de Desamparados, es importante generar un esbozo de las variables que integran ese riesgo (amenaza y vulnerabilidad), a partir de un estudio de las condiciones que hacen este territorio un crisol de procesos naturales tanto endógenos como exógenos, que podrían volverse peligrosos para la población. Por otro lado, se debe partir de la investigación de las características socioeconómicas como la pobreza, la calidad de la educación, el estado de la infraestructura pública y privada, la aplicación de las políticas nacionales y locales, así como la falta de un ordenamiento territorial efectivo, variables que favorecen la construcción de escenarios de riesgo en el municipio.

De acuerdo con la base de datos DesInventar (LA RED, 2016), Desamparados durante 1970 y el 2014 reportó un total de 835 eventos de desastre, convirtiéndose así en el cantón con el mayor número de reportes para ese periodo en Costa Rica. Esta aseveración coincide con otros estudios particulares por año (Brenes y Bonilla, 2005; Brenes y Bonilla, 2006; Brenes, Bonilla y Solís, 2007; Brenes y Bonilla, 2009; Brenes, 2012; Brenes, 2013; Brenes, 2014), así como con el de Arroyo (2011) entre el 2000 y el 2006. Según Quesada-Román (2015), a escala del Valle Central, durante 1985 y el 2013, las amenazas naturales más recurrentes fueron las inundaciones y deslizamientos en un $88 \%$ de los casos, tendencia clara para Costa Rica también. Esta situación se repite en Desamparados, donde las inundaciones y los deslizamientos (agregando también lluvias, avenida torrencial, aluvión y tormenta eléctrica) envuelven un $86 \%$ de los desas- tres reportados por DesInventar a lo largo de 44 años (Cuadro 4).

Cuadro 4. Cantidad de reportes por evento en Desamparados, según DesInventar, entre 1970 y el 2014

\begin{tabular}{|l|c|}
\hline \multicolumn{1}{|c|}{ Evento } & Cantidad de eventos \\
\hline Inundación & 423 \\
\hline Deslizamiento & 275 \\
\hline Incendio & 50 \\
\hline Vendaval & 42 \\
\hline Lluvias & 18 \\
\hline Avenida torrencial & 7 \\
\hline Sismo & 5 \\
\hline Colapso estructural & 4 \\
\hline Epidemia & 2 \\
\hline Explosión & 2 \\
\hline Plaga & 2 \\
\hline Sequía & 2 \\
\hline Aluvión & 1 \\
\hline Escape & 1 \\
\hline Tormenta eléctrica & 1 \\
\hline
\end{tabular}

Fuente: LA RED (2016).

Dos de los eventos por tomar en cuenta con una interesante proporción en el cantón fueron los incendios (tanto de casas de habitación como de lotes baldíos), así como los vendavales, los cuales son fenómenos meteorológicos relacionados con fuertes vientos que, al ser constantes y duraderos por lapsos considerables, pueden provocar afectación en la infraestructura, los cultivos, el tránsito vehicular y la salud. De los demás desastres reportados, solamente los sismos con cinco reportes y las sequías con dos tienen un origen natural, el resto de los eventos son de origen antrópico (colapso estructural, epidemia, explosión, plaga y escape). 
Del total de eventos históricos por distrito para Desamparados (Cuadro 5), entre tres de los 13 distritos del cantón se concentran $55.3 \%$ de reportes (Desamparados, San Miguel y Los Guido), los cuales suman el $43.3 \%$ del total de población para el 2011 (INEC, 2011). Esto a su vez tiene un reflejo en la densidad de población localizada en estos distritos y la localización de muchas viviendas en zonas de riesgo, debido a sus condiciones de vulnerabilidad económica, educativa, social e incluso institucional, por la falta de capacidad de respuesta ante este fenómeno en aumento. Es interesante apuntar que no menos despreciables son los datos que aportan distritos como Patarrá, San Juan de Dios, San Rafael Arriba y San Rafael Abajo, también con condiciones urbanas en una buena mayoría de sus habitantes y paisajes, lo que favorece la escorrentía del agua y procesos recurrentes como las inundaciones y encharcamientos.

Cuadro 5. Cantidad de eventos reportados por distrito en Desamparados, según DesInventar, entre 1970 y el 2014

\begin{tabular}{|l|c|}
\hline \multicolumn{1}{|c|}{ Distritos } & Cantidad de eventos \\
\hline Desamparados & 197 \\
\hline San Miguel & 164 \\
\hline Los Guido & 101 \\
\hline Patarrá & 74 \\
\hline San Juan de Dios & 67 \\
\hline San Rafael Arriba & 62 \\
\hline San Rafael Abajo & 57 \\
\hline San Antonio & 35 \\
\hline Rosario & 25 \\
\hline San Cristóbal & 21 \\
\hline Damas & 17 \\
\hline Frailes & 11 \\
\hline Gravilias & 4 \\
\hline
\end{tabular}

Fuente: LA RED (2016).
Los años que han reportado mayor cantidad de eventos se asocian de manera directa con los últimos 16 (1998-2014), se nota un salto en la cantidad de reportes de desastres a partir de 1998, cuando se pasó de un promedio de 4.5 eventos por año, entre 1970 y 1997, a 42.7 casos por año, entre 1998 y el 2014 (LA RED, 2016). Además, se debe hacer un claro reflejo sobre la densidad de población del cantón; ha llegado a superar incluso los 1000 habitantes por kilómetro cuadrado y la concentración de población bajo condiciones urbanas ha llegado a ser superior al $90 \%$ (INEC, 2011; Brenes, 2012).

Cuadro 6. Cantidad de reportes en los años de mayor recurrencia de impacto en Desamparados, según DesInventar, entre 1970 y el 2014

\begin{tabular}{|c|c|}
\hline Años & Cantidad de eventos \\
\hline 2008 & 118 \\
\hline 2007 & 83 \\
\hline 2005 & 64 \\
\hline 2004 & 60 \\
\hline 2003 & 59 \\
\hline 2011 & 48 \\
\hline 2010 & 44 \\
\hline 1999 & 43 \\
\hline 2013 & 41 \\
\hline 2006 & 38 \\
\hline
\end{tabular}

Fuente: LA RED (2016).

Como se observa en el Cuadro 6, el 2008 se asoció con un número mayor de reportes de desastres en Desamparados: 118 casos. Durante el mes de octubre de ese año, se presentó sobre Costa Rica un sistema de bajas presiones que afectó en gran medida el Valle Central, con mayor intensidad el municipio de Desamparados, para el cual 
fueron reportadas importantes afectaciones como inundaciones y deslizamientos, hubo al menos 16 evacuados y algunos sectores de la red vial cerrados, por lo que se dio una declaratoria de alerta roja para el cantón (CNE, 2008). En los restantes años los reportes de eventos de desastres se asocian con fuertes lluvias, temporales vinculados a sistemas de baja presión, y con saturación de alcantarillas (DesInventar, 2016).

Las condiciones de alta vulnerabilidad y exposición son principalmente el resultado de los sesgados procesos de desarrollo, incluyendo aquellos relacionados con la mala gestión del medio ambiente, los cambios demográficos, la urbanización rápida y no planificada, y la escasez de opciones de medios de vida para los grupos sociales desfavorecidos (Cardona et al., 2012). El caso de Costa Rica no dista de esta realidad, lo que se refleja en municipios y comunidades, aumentando la probabilidad de que procesos naturales se vuelvan peligrosos en entornos especialmente urbanos. De acuerdo con Martínez (2015), Desamparados, dentro de su marco regional que es la Gran Área Metropolitana (GAM), ha padecido de severos cambios ambientales, entre ellos, altas tasas de deforestación en la década de 1970. Cuando el Plan GAM de 1982 entró a regir, ya existía un panorama de seria afectación ambiental, deterioro de los recursos naturales y reducción de la cobertura vegetal; no obstante, la definición del Anillo de Contención urbana y la Zona Especial de Protección ayudaron a mitigar el impacto y la velocidad de esta problemática. Además, según el mismo autor, el área construida de la GAM aumentó a un ritmo acelerado (235 $\%$ entre 1980 y el 2000), con un crecimiento desordenado de la mancha urbana, fundamentalmente, lineal y centrífugo (en forma radial concéntrica o tentacular), siguiendo la red vial.
La población neta del cantón también experimentó en escasas cuatro décadas una explosión demográfica, que, de acuerdo con los censos de población de 1973, 1984, 2000 y 2011, pasa de 74272 a 208411 habitantes, lo que significó un aumento del 280 \% (CCPUCR, 2014). Esta condición demográfica fue producto, entre otras cosas, de la inmigración del campo a la ciudad, mejores condiciones en salud pública, mayores ofertas de empleo y la centralización de la institucionalidad costarricense en educación y diversos servicios del Estado. Este crecimiento atenuado de la población de Desamparados fue de la mano de la urbanización de los usos de la tierra, ya que se pasó de extensas áreas de cultivo, bosques y pastos a zonas urbanas. De acuerdo con los datos del INEC, en los censos de población y vivienda, el municipio pasa de tener un $86.6 \%$ de población urbana, en 1984, a un $93.6 \%$, para 2011; situación que atenúa la condición de suelos impermeabilizados, que modifican la escorrentía superficial y favorecen los procesos de inundación en regiones densamente pobladas.

Según el INEC (2011), entre los distritos más densamente poblados de Desamparados resaltan Desamparados (central) y San Rafael Abajo, con valores superiores a 8000 habitantes por kilómetro cuadrado, seguidos por San Juan de Dios, San Rafael Arriba, San Antonio, Damas, Gravilias y Los Guido, con rangos entre 4000 y $8000 \mathrm{hab} / \mathrm{km} 2$; mientras que San Miguel, Frailes, Patarrá, San Cristóbal y Rosario no superan los 4000 hab/km2. Por tanto, dicha concentración poblacional muchas veces va de la mano con situaciones de limitación económica y pobreza, reflejadas en el $19.4 \%$ de la población de Desamparados, que para el 2011 no tenía satisfechas sus necesidades básicas como albergue, salud, conocimiento y consumo; estos grupos sociales tienen poca resiliencia o capacidad 
de adaptarse a escenarios de riesgo, lo que conlleva problemáticas sociales peores como la pérdida de viviendas, la vida humana y el modo de vida. Desamparados presenta de las tasas proyectadas más altas de crecimiento poblacional y de los porcentajes más elevados de déficit de vivienda, por lo cual se consolida como lugar de residencia para hogares de ingresos medio-bajos y bajos. A su vez, esto muestra serios requerimientos para solventar sus necesidades actuales y futuras, lo que va de manera paralela al desarrollo de condominios horizontales de alto poder adquisitivo (Martínez, 2015).

La política pública tiene la capacidad de regular la sociedad, donde claramente existirán diferencias de intereses sociales, económicos, ambientales e incluso políticos; no obstante, debe encontrarse un equilibrio entre los agentes involucrados (Calderón-Ramírez, 2015). La ineficiencia en el ordenamiento territorial origina que exista un crecimiento urbano desordenado, sin planificación en temas de redes, vialidad, límite de construcción, entre otros. Por las características morfológicas que presenta el cantón de Desamparados y según el registro de eventos naturales, se le debe dar una atención y pronta respuesta. Al no tener un reglamento que regule el crecimiento de los asentamientos urbanos, se generan áreas con alta vulnerabilidad de riesgo de desastre, debido a que las construcciones de estas se desarrollan en áreas no aptas para urbanizar. Las políticas de ordenamiento territorial no han tenido verdadero impacto en la planificación del espacio de Desamparados, debido a que, a pesar de que existe una serie de leyes y reglamentos (algunos obsoletos), no hay una ley nacional de ordenamiento territorial propiamente; además, el plan regulador no contempla su contexto regional (urbano ni rural) ni de cuenca hidrográfica, y su vialidad ambiental (índices de fragilidad ambiental: IFA) es definida por metodologías que son confusas y no representan la realidad del cantón (Barrantes, 2012; Barrantes, 2013). Estas disparidades en la planificación del territorio de Desamparados, además de generar un aumento en la ocurrencia de desastres, han generado, a lo largo de las últimas cuatro décadas, problemáticas como la contaminación ambiental (en aire, agua, residuos sólidos y las emisiones de gases de efecto de invernadero), el colapso de la vialidad (tanto en vías principales como periféricas del cantón), el crecimiento urbanístico desordenado sin áreas verdes ni recreativas, la especulación en el mercado de tierras, la concentración de focos de pobreza y, por ende, la inseguridad en zonas marginales (La Capri, Calle Fallas, Los Guido, Patarrá, entre otros).

\section{Discusión}

Se han realizado importantes avances en los últimos años hacia una estructuración de la legislación sobre ordenamiento territorial y gestión del riesgo de desastres en Costa Rica y la Gran Área Metropolitana (GAM) (región que alberga el $60 \%$ de la población del país), por medio de la creación de la Política Nacional de Ordenamiento Territorial (PNOT), el Plan Nacional de Ordenamiento Territorial (PLANOT), el Plan de Ordenamiento Territorial del Gran Área Metropolitana (PLAN GAM 2013), además de la Ley Nacional de Emergencia 7914 y la Ley Nacional de Emergencias y Prevención del Riesgo. 8488; políticas aplicables a las escalas nacional, regional y local, en las que se busca la integración de todas las instituciones públicas encargadas de la planificación del territorio, de acuerdo con sus funciones particulares. No obstante, 
la ausencia en la actualidad de un ordenamiento territorial efectivo y la integración de la variable de gestión del riesgo de desastres en Costa Rica es un elemento más en la fórmula que conforma el riesgo del país. A esto deben sumarse factores como el crecimiento del porcentaje de población urbana vs. el de la rural, así como la presión urbanística, el cambio en el uso de la tierra, la demanda de servicios e infraestructura pública y privada, la vulnerabilidad hídrica (contaminación de aguas superficiales y subterráneas), al igual que el desarrollo de anillos de pobreza y tugurios.

Para mejorar las condiciones de riesgo del país, se deben tomar medidas desde las instituciones públicas y privadas, en todos los estratos sociales, tanto en la ruralidad como en la urbanidad, así como en los distintos niveles educativos. Se requieren mejores estudios y cartografías, a escalas detalladas, que sean avalados por la Comisión Nacional de Prevención de Riesgos y Atención de Emergencias (CNE) y generados del vínculo con las universidades públicas y expertos en la temática. En esta misma línea, se debe procurar crear más estudios sobre multiamenazas, ligados a análisis probabilísticos de acuerdo con las características particulares de las cuencas que integran el país. Además, todos estos insumos deben ser divulgados en diferentes grupos y estratos sociales, tanto en las comunidades urbanas como en las rurales; en el nivel escolar, secundario y universitario, entre sus planes de estudio.

No debe desestimarse o mermarse la inversión en los centros de investigación y monitoreo como el Observatorio Vulcanológico y Sismológico de Costa Rica (OVSICORI), la Red Sismológica Nacional (RSNICE-UCR), el Instituto Meteorológico Nacional (IMN), así como la Comisión Na- cional de Prevención de Riesgos y Atención de Emergencias (CNE), la cual coordina la promoción y ejecución de los lineamientos de política pública que permiten al Estado costarricense y a los distintos sectores de la actividad nacional incorporar la gestión del riesgo como eje transversal en la planificación y el desarrollo del país. Se debe mejorar la generación de información sobre las amenazas y vulnerabilidades sociales en la medida que se integran con la gestión del riesgo; por tanto, es importante realizar estudios y políticas que indiquen los diferentes tipos de debilidades físicas, sociales, económicas, ambientales y globales que tienen tanto el territorio como la población nacional y de la Gran Área Metropolitana. Es fundamental el desarrollo de cartografía a detalle de la vulnerabilidad, para lograr el análisis preciso del riesgo que tiene el país en términos micro- y macroeconómicos. A la par de estos estudios detallados, se debe hacer una inversión aún mayor en verdadera gestión del riesgo de desastres, que permita disminuir la exposición, reducir el peligro y mejorar la adaptación al cambio climático y los riesgos naturales, creando mayor resiliencia y evitando que las pérdidas económicas aumenten año tras año.

Las amenazas naturales están presentes todos los años en Costa Rica, donde la dinámica tectónica, geológica, geomorfológica e hidrometeorológica se mantiene en constante cambio a diferentes escalas geográficas, magnitudes e intensidades. Las inundaciones y deslizamientos son los procesos más recurrentes y causan millones de dólares en pérdidas económicas cuando se convierten en desastres, en todas las esferas del país: educación, salud, comercio, infraestructura pública y privada, y en la vida humana. Entre 1988 y el, 2009 el país tuvo pérdidas económicas relacionadas, de manera directa e indirecta, 
con desastres, por 1823 millones de dólares estadounidenses. El mayor número de tipos de eventos fueron los hidrometeorológicos con $34(82,9 \%)$; los sismos representaron el 12,2\%. El sector público es el más afectado por el impacto de desastres, un $62 \%$ del total de eventos que generaron pérdidas económicas se relaciona con infraestructura pública $\mathrm{y}$, en este rubro, se consideraron el MOPT, el Ministerio de Salud, Acueductos Rurales y Urbanos, Edificaciones Públicas y Ferrovías. Las pérdidas promedio representaron un $0,83 \%$ del producto interno bruto (PIB) por año, durante el periodo 2005-2009, situación que en algunos años aumenta hasta casi alcanzar el $2 \%$ del PIB (MIDEPLAN, 2010).

Durante el 2005 y el 2011, las pérdidas ocasionadas por los fenómenos naturales que merecieron declaratoria de emergencia se estimaron en 1130,09 millones de dólares, lo que arroja un promedio anual de poco más de 188 millones de dólares (al precio del dólar estadounidense en el 2011). Para ese periodo, resaltan varios temporales, el impacto directo e indirecto de diversos ciclones tropicales (69 $\%$ del total) y el terremoto de Cinchona, lo que incidió en procesos de inundación, deslizamientos, destrucción de infraestructura pública y privada como viviendas, líneas vitales y vidas humanas (MAG-MIDEPLAN, 2013). Además de esto, una vez suscitado el fenómeno natural, se da una desviación de recursos orientados a prioridades productivas y de desarrollo para la reparación y rehabilitación de los daños producidos por los desastres (MIDEPLAN, 2010). A esta situación debe sumarse la acumulación de las pérdidas económicas por desastres a lo largo del tiempo, la cual se estima en unos 86 millones de dólares estadounidenses en promedio, al año, cifra que es aún más preocupante por su efecto acumulativo a lo largo del tiempo, lo que reduce la capacidad de respuesta del país y limita el desarrollo local, regional y nacional (Astorga, 2010). Más preocupante aún es el desconocimiento de los impactos económicos asociados a desastres que ocurren todos los años y no son cuantificados por los entes gubernamentales locales; pérdidas económicas que afectan la propiedad pública y privada y no son objeto de planes de reparación, debido a que se hace hincapié y mayor análisis, en términos monetarios, a eventos con magnitudes e intensidades apreciables en el contexto regional o nacional (Quesada-Román, 2015).

Existe una clara tendencia de crecimiento, para las siguientes décadas, de las afectaciones económicas debido a desastres en Costa Rica. Al tener en cuenta que la mayor cantidad de impactos y pérdidas económicas son de tipo hidrometeorológico, cabe tomar medidas esclarecedoras por parte del Estado costarricense ante el cambio climático, además de definir políticas claras de reducción de la vulnerabilidad ante los desastres, desde las escalas nacional, regional y local, que vinculen las diversas instituciones públicas y privadas involucradas en esta temática (Adamson-Badilla, 2012). Las proyecciones de cambios en el clima en las siguientes décadas indican un aumento en los periodos secos asociados al fenómeno del Niño, en descensos de la precipitación y aumentos de la temperatura, pero, a su vez, se anuncian años con precipitaciones más intensas en periodos más cortos que podrían generar mayores inundaciones y cargar las cuencas hidrográficas, llenando la capacidad de campo de sus suelos y provocando un número creciente de deslizamientos. Cabe resaltar que los cambios en el clima son un hecho y deben ser trabajados como ejes transversales en conjunto con la gestión del riesgo. 


\section{Conclusiones}

Dada su localización en un contexto tectónico, geológico y geomorfológico complejo y geodinámicamente activo, Desamparados posee un espacio geográfico donde los deslizamientos e inundaciones son las amenazas naturales más recurrentes, hecho que lo constituye como como uno de los municipios más afectados por desastres cada año, lo que, a su vez, se traduce en pérdidas económicas y de vidas humanas. Sin embargo, no existen estudios de las amenazas naturales del cantón a escalas detalladas (1:25.000, 1:10.000 o superiores), por lo que se debe procurar el desarrollo de más indagaciones sobre multiamenazas, ligados con análisis probabilísticos acordes con las características particulares de las cuencas hidrográficas que integran el municipio. Las investigaciones de cambio climático y gestión del riesgo deben ser ejes transversales para las diferentes esferas de toma de decisiones en los municipios como Desamparados, donde estas dos variables sean tomadas en cuenta desde la educación, la salud pública, la formulación de proyectos comunales, el desarrollo de nueva infraestructura pública, el medio ambiente y el ordenamiento territorial.

Es fundamental, en el nivel nacional, la creación de una ley de ordenamiento territorial que integre todos los elementos de planificación de los municipios, desde las escalas nacional, regional y local, contemplando las cuencas hidrográficas, la Zona Marítimo Terrestre y la Gestión del Riesgo de Desastres como principales ejes transversales. En el caso de Desamparados, se debe mejorar la acción municipal respecto a los desastres, para crear una oficina de gestión de riesgo que genere una conciencia más amplia sobre la problemática del desastre local, ejemplo que debería aplicarse también para el resto de los municipios de Costa Rica. A Desamparados, por sus características físico-geográficas y poblacionales, se le debe prestar gran atención a futuro, en función de cómo se están manejando las políticas públicas respecto a la gestión del riesgo de desastres.

Las condiciones socioeconómicas de la población del cantón de Desamparados crean una influencia negativa que favorece la vulnerabilidad a riesgos del desastre. En algunos casos, la falta de recursos económicos y la necesidad de tener una vivienda hacen que la población de este sector desarrolle un crecimiento urbano sin planificación a orillas de ríos o zonas vulnerables. Lo anterior se une a la falta de una política e intervención estatal en tema de ordenamiento territorial. Política es sinónimo de poder, por ende, es claro que se debe intervenir en el territorio y claramente en el tema de discusión. Lo anterior se abona a la falta directa en la gestión local del riesgo, la cual le compete a la municipalidad, y es de conocimiento que no existe una ley que dicte a los gobiernos locales que deben tener un profesional quien intervenga de manera inmediata en la gestión del riesgo de desastres.

\section{Referencias}

Adamson-Badilla, M. (2012). Desastres y desarrollo en Costa Rica. En M. Adamson, M. y F. Castillo (eds.), Desastres: Costa Rica en el tercer milenio desafios y propuestas para la reducción de vulnerabilidad. San José, C. R.: Contrastes Vivos de Costa Rica.

Alfaro, E. y Quesada-Román, A. (2010). Ocurrencia de ciclones tropicales en el Mar Caribe y sus impactos sobre Centroamérica. InterSedes, XI(22-2010), 136-153. ISSN: 2215-2458.

Arroyo, L. N. (2011). Costa Rica: análisis de la incidencia espacial de inundaciones y deslizamientos por provincias y cantones, años 2000-2006. Revista Geográfica de América Central, 47, 97-126. 
Astorga, A. (2010). Ordenamiento territorial en Costa Rica. Decimoséptimo Estado de la Nación. Consejo Nacional de Rectores.

Azevedo, J. M. (1997). A Educação como Política Pública. Campinas: SP: Autores Associados.

Barrantes, G. (2012). Deficiencias del Índice de Fragilidad Ambiental en la valoración de las amenazas naturales para la planificación territorial. En torno a la Prevención, 9.

Barrantes, G. (2013). Deficiencias del Índice de Fragilidad Ambiental en la valoración de las amenazas naturales para la planificación territorial: Parte II: Propuesta metodológica para su corrección. En torno a la Prevención, 11.

Bird, P. (2003). An updated digital model of plate boundaries. Geochemistry, Geophysics, Geosystems, 4(3): 1-52. Doi https://doi.or$\mathrm{g} / 10.1029 / 2001 \mathrm{GC} 000252$

Brenes, A y Bonilla, A. (2006). Gestión del riesgo. Duodécimo Estado de la Nación en Desarrollo Humano Sostenible. Consejo Nacional de Rectores.

Brenes, A. (2012). Gestión del riesgo. Decimoctavo Estado de la Nación en Desarrollo Humano Sostenible. Consejo Nacional de Rectores.

Brenes, A. (2013). Gestión del riesgo. Decimonoveno Estado de la Nación en Desarrollo Humano Sostenible. Consejo Nacional de Rectores.

Brenes, A. (2014). Gestión del riesgo. Vigésimo Estado de la Nación en Desarrollo Humano Sostenible. Consejo Nacional de Rectores.

Brenes, A. y Bonilla, A. (2005). Gestión del riesgo. Undécimo Estado de la Nación en Desarrollo Humano Sostenible. Consejo Nacional de Rectores.

Brenes, A. y Bonilla, A. (2009). Gestión del riesgo y cambio climático. Decimoquinto Estado de la Nación en Desarrollo Humano Sostenible. Consejo Nacional de Rectores.

Brenes, A.; Bonilla, A. y Solís, A. (2007). Gestión del riesgo. Decimotercer Estado de la Nación en Desarrollo Humano Sostenible. Consejo Nacional de Rectores.

Calderón-Ramírez, G. (2015). Costa Rica: Um estudo geográfico da pobreza e a contribuição das políticas públicas. Brasil: Universidade Federal de Rio Grande.

Cardona, O. D.; van Aalst, M. K.; Birkmann, J.; Fordham, M.; McGregor, G.; Perez, R.; Pulwarty, R. S.; Schipper, E. L. F. y Sinh, B. T. (2012). Determinants of risk: exposure and vulnerability. En C. B. Field, V. Barros,
T. F. Stocker, D. Qin, D. J. Dokken, K. L. Ebi, M. D. Mastrandrea, K. J. Mach, G. -K. Plattner, S. K. Allen, M. Tignor y P. M. Midgley (eds.), Managing the Risks of Extreme Events and Disasters to Advance Climate Change Adaptation. A Special Report of Working Groups I and II of the Intergovernmental Panel on Climate Change (IPCC) (pp. 65108). Cambridge y Nueva York: Cambridge University Press. Doi https://doi.org/10.1017/ CBO9781139177245.005

CCP-UCR-Centro Centroamericano de Población, Universidad de Costa Rica. (2014). InfoCensos (17 de junio de 2016). Recuperado de http://infocensos.ccp.ucr.ac.cr

Comisión Nacional de Prevención de Riesgos y Atención de Emergencias (CNE). (2008). Evento: inundaciones y deslizamientos por influencia de sistemas de bajaa presiones sobre territorio costarricense. Informe de situación No. 3. martes 14 de octubre del 2008.

DeMets, C.; Gordon, R. G.; Argus, D. F. y Stein, S. (1990). Current plate motions. Geophysics. J. Int., 101, 425-478. Doi https://doi.org/10.1111/j.1365-246X.1990.tb06579.x

Denyer, P.; Montero, W. y Alvarado, G. E. (2003). Atlas tectónico de Costa Rica. San José, C. R.: Editorial de la Universidad de Costa Rica.

EIRD-ONU (Estrategia Internacional para la Reducción de Desastres). (2016). Terminología: Términos principales relativos a la reducción del riesgo de desastres. Recuperado de http:// www.eird.org/esp/terminologia-esp.htm

IMN-Instituto Meteorológico Nacional (2008). Atlas Climático Interactivo. San José, C. R.

INEC-Instituto Nacional de Estadística y Censos (2011). Resultados generales de población y vivienda del Censo 2011. San José, C. R.

LA RED. (2016). Base de datos de desastres DesInventar. Recuperado de http://online.desinventar.org/

MAG-Ministerio de Agricultura y Ganadería; MIDEPLAN-Ministerio de Planificación Nacional y Política Económica. (2013). Sistematización de la Información de Impacto de los Fenómenos Naturales en Costa Rica, Período 2005-2011. Costa Rica, noviembre, 2013.

Martínez, T. (2015). Treinta años de Metamorfosis Urbana Territorial en el Valle Central. Informe Final. Vigesimoprimer Informe Estado de la Nación en Desarrollo Humano Sostenible (2014). 
MIDEPLAN-Ministerio de Planificación Nacional y Política Económica. (2010). El impacto económico de los eventos naturales y antrópicos extremos en Costa Rica, 1988-2009. Costa Rica, octubre, 2010.

MIDEPLAN-Ministerio de Planificación Nacional y Política Económica. (2012). Área de Análisis del Desarrollo de Objetivos de desarrollo del milenio (ODM) censo 2011: una aproximación cantonal. San José, C. R.

MIDEPLAN-Ministerio de Planificación Nacional y Política Económica de Costa Rica. (2013). Índice de Desarrollo Social 2013. San José, C. R.

PNUD-Programa de las Naciones Unidas para el Desarrollo. (2007). Atlas del desarrollo humano cantonal de Costa Rica 2007. San José, C.R: Universidad de Costa Rica. Recuperado el 12 de junio de 2016 de http://www.pnud.or.cr/ dmdocuments/ATLAS2007.pdf.

PNUD-Programa de las Naciones Unidas para el Desarrollo. (2011). Atlas del desarrollo humano cantonal de Costa Rica 2011. San José, C.R: Universidad de Costa Rica. Recuperado de http://www.pnud.or.cr/mapa-cantonal/atlas. pdf

Programa Estado de la Nación. Decimosexto Informe Estado de la Nación en Desarrollo Humano Sostenible, 2010. Capítulo 4. Armo- nía con la naturaleza. Recuperado de http:// www.estadonacion.or.cr/files/biblioteca_virtual/016/4-CAP-4-informe16.pdf

Quesada-Román, A. (2015). Implicaciones en la gestión del riesgo de desastres y ambiente en el Valle Central en los últimos treinta años (1985-2015). Vigesimoprimer Informe Estado de la Nación en Desarrollo Humano Sostenible (2014).

Ramírez, A. y Villalobos, M. (2014). Marco normativo, institucionalidad y conflictividad del ordenamiento territorial. Vigesimoprimer Informe Estado de la Nación en Desarrollo Humano Sostenible (2014).

Souza, C. (2006). Políticas Públicas: uma revisão da literatura. Sociologias Porto Alegre, 16, 20-45. Doi https://doi.org/10.1590/S151745222006000200003

Taylor, M. y Alfaro, E. (2005). Climate of Central America and the Caribbean. En J. Oliver (ed.), Encyclopedia of world climatology. Berlín: Springer. Doi https://doi.org/10.1007/14020-3266-8_37

Vargas, G. (2012). Geografia de Costa Rica. San José, C. R.: Editorial EUNED.

\section{(9) $\odot \Theta \Theta$}

Gestión del riesgo y política pública en el cantón de Desamparados, Costa Rica (Adolfo Quesada-Román y Guillermo Calderón-Ramírez) por Revista Uniciencia se encuentra bajo una Licencia CreativeCommons Atribución-NoComercial-SinDerivadas 3.0 Unported. 